\title{
A implementação da Terapia Comunitária Integrativa em uma unidade de saúde e seu auxílio no cuidado integral: um relato exitoso
}

\section{The implementation of Integrative Community Therapy in a health unit and its assistance in integral care: a successful report}

\author{
Morgana Pordeus do Nascimento Forte ${ }^{1}$. Andréa de Souza Gonçalves Pereira ${ }^{2}$. \\ 1 Universidade Federal do Ceará (UFC), Universidade de Fortaleza (UNIFOR), Fortaleza, Ceará, Brasil. 2 Secretária Municipal \\ de Saúde de Fortaleza, Fortaleza, Ceará, Brasil.
}

\section{RESUMO}

Objetivos: Descrever a implementação da Terapia Comunitária Integrativa (TCI) na Unidade Atenção Primária à Saúde (UAPS) Edmar Fujita do município de Fortaleza - Ceará. Métodos: Análise descritiva da TCI como prática de promoção e atenção à saúde mental e integral na Atenção Primária (AP) e revisão da literatura sobre o tema. Resultados: A TCI vem sendo realizada em um turno semanal, cujas facilitadoras foram capacitadas pelo Projeto Quatro Varas. É praticada em ambiente dentro da comunidade e é aberto ao público. Observa-se aumento do vínculo com a equipe promotora, bem como uma facilitação na coordenação do cuidado de maneira integral. É evidente a maior demanda de atendimentos médicos voltados aos participantes, os quais não necessariamente são da área de abrangência da UAPS. Conclusões: A TCI objetiva a promoção e criação de redes de apoio para lidar com demandas psicossociais. É um instrumento pouco oneroso que promove melhorias quanto à coordenação do cuidado, principalmente daqueles pertencentes à área adscrita da UAPS, aumentando assim o poder de resolução da AP. Foi possível observar maior demanda de atendimentos em saúde mental, o que reforça a necessidade da implantação de práticas integrativas como a TCI como ferramenta para um plano terapêutico individual e comunitário.

Palavras-chave: Saúde mental. Sistema Único de Saúde. Terapias complementares. Atenção primária à saúde.

\section{ABSTRACT}

Objectives: To describe the implementation of Integrative Community Therapy (ICT) in the Primary Health Care Unit (PHCU) Edmar Fujita of the city of Fortaleza - Ceará. Methods: Descriptive analysis of the ICT as a practice of promotion and attention to mental and integral health in Primary Care (PC) and review of the literature on the subject. Results: The ICT has been carried out in a weekly shift, whose facilitators were trained by the Quatro Varas Project. It is practiced in an environment within the community and is open to the public. It is observed an increase in the bond with the promoter team, as well as a facilitation in the coordination of the care of integral way. The demand for medical care for the participants is clearly higher, which is not necessarily within the scope of the UAPS. Conclusions: ICT aims to promote and create support networks to deal with psychosocial demands. It is an inexpensive instrument that promotes improvements in the coordination of care, especially those belonging to the area assigned to the PHCU, thus increasing the power of resolution of the PC. It was possible to observe a greater demand for mental health care, which reinforces the need to implement integrative practices such as ICT as a tool for an individual and community therapeutic plan.

Keywords: Mental health. Unified Health System. Complementary therapies. Primary health care.

Autor correspondente: Morgana Pordeus do Nascimento Forte, Rua Capitão Francisco Pedro, 1290, Rodolfo Teófilo, Fortaleza, Ceará. CEP: 60430372. Telefone: +55 85 98866-9959. E-mail: morganapordeus@gmail.com

Conflito de interesses: Não há qualquer conflito de interesses por parte de qualquer um dos autores.

Recebido em: 16 Set 2018; Revisado em: 22 Nov 2018; Aceito em: 09 Jan 2019. 


\section{INTRODUÇÃO}

No Brasil, pela Constituição Federal de 1988, reconheceuse a saúde como um direito fundamental e um dever do Estado, a qual, conceitua-se de acordo com a Organização Mundial de Saúde (OMS), como o estado do mais completo bem-estar físico, mental e social e não apenas a ausência de enfermidade. ${ }^{1,2}$

Com intuito de garantir a saúde no País, foi criado o Sistema Único de Saúde (SUS), o qual tem como alguns de seus princípios a universalidade, a integralidade, a equidade, a descentralização e a participação da comunidade. Além de estar organizado em três níveis de atenção, sendo a Atenção Primária um deles, voltada para práticas de promoção, prevenção, manutenção e reabilitação de saúde. . $^{3,4}$

A localização estratégica da Atenção Primária, permite que o processo saúde-doença seja abordado em diversas instâncias, a partir da criação de vínculos de responsabilidade entre os sujeitos comunitários, do diálogo, da espiritualidade, da ressalva da posição familiar e de considerar o contexto social como norteador para ações de saúde, substituindo o modelo tradicional e levando a saúde para mais perto da família.5,6,7

A OMS e a Organização Panamericana de Saúde (OPAS) consideram a saúde mental como uma prioridade dos governos latino-americanos, já que o modo de vida das pessoas vem mudando significativamente com a modernização e é evidente o aumento da desigualdade e exclusão em sociedades capitalistas, sendo estas contribuintes para o processo de adoecimento mental, o qual, muitas vezes, só é percebido após desencadear sintomas físicos e levar as pessoas à procura por serviços de saúde na busca por medicação que aliviem o sofrimento. ${ }^{7,8}$

Nesse contexto, as Práticas Integrativas e Complementares (PIC's) podem ser inseridas, pois envolvem abordagens que visam estimular os mecanismos naturais de prevenção de agravos e recuperação da saúde por meio de tecnologias eficazes e seguras, com ênfase na escuta acolhedora, no desenvolvimento do vínculo terapêutico e na integração do ser humano com o meio ambiente e a sociedade. ${ }^{8,9}$

No Brasil, a Política Nacional de Práticas Integrativas e Complementares (PNPIC) foi iniciada em 2003 no SUS, e aprovada em fevereiro de 2006 pela OMS, objetivando desenvolver uma sistemática de Atenção Básica, que auxilia de forma complementar os tratamentos médicos, proporcionando ao paciente uma nova forma terapêutica, menos invasiva a partir de estímulos e desenvolvimentos de promoção, prevenção e recuperação da saúde, utilizando-se métodos naturais como recurso, embasados no acolhimento e na criação de vínculos entre usuário, família e profissional de saúde..$^{9,10,11}$

Considerando que a OMS preconiza o reconhecimento e incorporação das Medicinas Tradicionais e Complementares, também conhecidas como PIC's, nos sistemas nacionais de saúde, a Terapia Comunitária Integrativa (TCI) se enquadra como recurso para lidar com demandas psicossociais e promover redes de apoio social, ampliando a resolutividade da Atenção Primária.

A TCI foi criada pelo Professor Doutor Adalberto de Paula Barreto, antropólogo e médico psiquiatra, a partir de um projeto de pesquisa e extensão na área da saúde comunitária, vinculada à Universidade Federal do Ceará, representando um espaço de escuta e acolhimento comunitário, por meio de troca de experiências. ${ }^{12}$

A TCI é um instrumento pertencente às PIC's que desponta como uma tecnologia de cuidado que tem dado respostas satisfatórias aos que dela se beneficiam, sendo mais um instrumento de trabalho, que pode ser utilizado pelos profissionais de saúde, áreas afins e pela própria comunidade. Está implantada em vários estados brasileiros, a partir das experiências e dos cursos para formação de terapeutas comunitários, e presente em dezenove países, dentre os quais é citado Suíça, Itália e França. Quando inserida na Atenção Primária, favorece a ampliação do vínculo entre profissional e comunidade. ${ }^{3,13}$

O presente artigo visa descrever como relato de experiência, a implementação da TCI na UAPS Edmar Fujita do município de Fortaleza - Ceará, bem como avaliar as consequências quanto a coordenação do cuidado após implantação.

\section{MÉTODOS}

O estudo foi realizado na UAPS Edmar Fujita, localizada no bairro da Boa Vista, no município de Fortaleza, local cuja autora principal atuou como residente em Medicina de Família e Comunidade (MFC) pela UFC no período de abril de 2016 a março de 2018 .

Para produção do artigo, além da análise qualitativa da implementação da TCI em uma unidade de saúde, buscou-se referências relevantes de idioma português para corroborar com a análise descritiva em bases de dados como LILACS, PubMed e Scielo, no período de outubro de 2017 a janeiro de 2018, utilizando os seguintes Descritores em Ciências da Saúde (DeCS): Terapia Comunitária; Atenção Primária à Saúde; Práticas Integrativas e Complementares.

A implementação da TCI foi realizada após capacitação de duas profissionais do serviço, pelo Movimento Integrado de Saúde Mental Comunitária (MISMEC - CE), sendo uma médica pertencente a uma equipe da UAPS e uma educadora em saúde vinculada à Secretária Municipal de Saúde de Fortaleza. A partir daí, foi escolhido e fixado um turno por semana para a atividade aberta ao público, já que a proposta da TCI é de que se ocorra com tal frequência, assim como é realizada na própria instituição de origem, o projeto Quatro Varas. Cada encontro dura cerca de duas horas e se desenvolve a partir das seguintes fases: acolhimento, escolha do tema, contextualização, problematização e encerramento, conforme orientado durante capacitação das terapeutas. $\mathrm{O}$ processo de 
capacitação das terapeutas se deu em oito módulos do curso de TCI, no período de agosto a dezembro de 2016, realizado pela MISMEC - CE, popularmente conhecido como Projeto Quatro Varas. Durante a capacitação, houve vinte encontros, sendo doze blocos teórico-práticos e oito estágios supervisionados. No período, já havia mobilização da UAPS Edmar Fujita para a implantação, por meio de reuniões com os profissionais de saúde para mostrar a importância de tal prática na comunidade. A partir de novembro, foi selecionado o turno da manhã de todas as quartas-feiras para iniciar as sessões de TCI.

\section{RESULTADOS}

O local escolhido para a prática da TCI foi a casa de um dos líderes comunitários, morador da comunidade há mais de 50 anos, sendo conhecido pela maioria da população da área. Foram realizados painéis informativos afixados na UAPS, palestras oportunas durante salas de espera para consultas agendadas ou demandas espontâneas, pelo menos uma vez na semana, realizadas por alunos de graduação da Universidade de Fortaleza (UNIFOR), pela própria residente em MFC, bem como pelas terapeutas capacitadas. Além disso, folhetos propagando a TCI foram impressos e disponibilizados nas salas da UAPS para melhor divulgação entre os próprios funcionários, bem como para que fossem distribuídos durante consultas eletivas individuais, nas quais eram feitos convites para participar da terapia, comunicando ser uma atividade gratuita, aberta ao público, sem faixa etária específica, na qual toda a família e/ou vínculos sociais poderiam participar.

Cada encontro da TCI se desenvolve em cinco etapas conforme descrito anteriormente: a primeira, o acolhimento, tem intuito de os participantes se conhecerem e estreitarem os laços, por meio de músicas, textos e atividades recreativas curtas trazidas pelos próprios participantes ou pelas terapeutas. Em seguida, ocorre a escolha do tema: abre-se espaço para quem deseja falar brevemente sobre aquilo que está lhe inquietando. Em ocorrendo mais de um tema, é eleito por votação apenas um, deixando claro que os temas não selecionados não são menos importantes. Após, ocorre a contextualização, em que o participante cujo tema foi escolhido descreve com mais detalhes a sua inquietação. Em seguida, com a problematização, questiona-se se alguém presente tem uma experiência semelhante e como foi capaz de lidar com tal adversidade. Por fim, o encerramento é o momento em que os próprios participantes revelam em que o encontro da TCI lhe foi útil.

Inicialmente, os participantes eram aqueles que possuíam mais vínculos com as terapeutas, porém ao longo das sessões a comunidade foi reconhecendo o espaço como mais uma oportunidade para articular redes de apoio, tecendo laços e propagando a TCI para além da localização da comunidade.

Observavam-se que o número de frequentadores variava, já que era uma atividade aberta ao público, chegando no máximo a 20 participantes, predominantemente mulheres, além de usuários vindos de outros bairros, trazendo demandas que não foram assistidas em alguns serviços de saúde.
Por uma das terapeutas ser médica, aqueles participantes que apresentavam sinais ou sintomas de transtornos mentais moderados a graves eram convocados para atendimento individual, principalmente quando não recebiam assistência até aquele momento. $\mathrm{Na}$ ocasião, costumava-se fazer uma escuta ativa inicial, manejar o transtorno mental e realizar o encaminhamento para a UAPS de abrangência. Em não sendo possível, o tratamento se mantinha na UAPS na qual a TCI estava sendo promovida.

Apesar de um dos objetivos da implementação da TCI ser o cuidado com usuários portadores de sofrimento mental, notou-se que ao longo das sessões, alguns usuários com outras comorbidades, como hipertensão, diabetes, osteroartrose, também utilizavam daquele espaço para partilhar experiências de suas condições clínicas e, ao final, tirar dúvidas referentes aos seus tratamentos com os profissionais participantes, principalmente a médica.

É possível compreender a TCI como uma prática complementar e que dá autonomia à comunidade para se reconhecer responsável pelo próprio cuidado, mas em alguns momentos tal prática servia como acolhimento e encaminhamento para os serviços de saúde, enfatizando a importância de a mesma ser conduzida e/ou frequentada por profissionais da Atenção Primária, já que a prática se torna também uma porta de entrada para o SUS.

É evidente que a TCI é capaz de facilitar o acesso e o vínculo com a equipe promotora, e se torna um auxílio para os profissionais de saúde no manejo de transtornos mentais ou clínicos, e corrobora para o plano terapêutico individual e coletivo.

Ao longo das percepções que as sessões de TCI trouxe, questionou-se o acolhimento em outros serviços de atenção primária à saúde, tamanha demanda de usuários de outras comunidades, principalmente no que se refere à saúde mental.

A possibilidade de tal prática ser obrigatória em outras UAPS do município deve ser considerada, pois pode ser realizada por qualquer profissional desde que seja capacitado, além de ser uma ferramenta de baixo custo que melhora a capacidade de resposta da Atenção Primária.

\section{DISCUSSÃO}

No Brasil, o SUS proporcionou avanços no campo das políticas públicas, desenvolvendo práticas diferentes do modelo tradicional de saúde. A integralidade, um de seus princípios, tem como perspectiva a não restrição das pessoas somente a condições que provocam sofrimento, mas reconhecimento e articulação de ações preventivas e assistenciais sobre a condição, principalmente através de diálogo. Nesse aspecto, a implementação da TCI contemplou uma forma de articular redes de apoio através de acolhimento e escuta ativa de demandas psicossociais, constituindo uma excelente forma de abordagem integral. ${ }^{14,15}$ 
O cotidiano dos brasileiros é marcado por problemas e sofrimentos que acarretam danos à sua saúde de um modo geral.É então nas UAPS's que o cuidado à saúde integral dos brasileiros transita entre modelos de enfoque individual e coletivo em busca de desenvolver ações de prevenção à doença mental e ao sofrimento psíquico. Para isso, os profissionais que atuam nessas equipes de saúde, devem estar capacitados em Saúde Mental, logo a TCI pode ser uma das formas de abordar um público cuja demanda psíquica é evidente. Com a implementação, observamos que os principais problemas trazidos eram tristeza, depressão, choro fácil, ansiedade, transtorno do sono, angústia, problemas familiares que acarretam dores físicas, etc., sendo, portanto, mais um instrumento de prevenção e promoção de cuidados em Saúde Mental. ${ }^{3,5,16}$

Os profissionais atuantes nas UAPS's devem estar capacitados para atender a diversos tipos de sofrimento. É descrito inclusive que as ações em saúde devem organizar-se dentro da lógica extramural e da lógica da reconstrução da cidadania plena, logo promover um espaço comunitário e acolhedor por meio da TCI na UAPS referida no artigo fez com que os profissionais terapeutas atuantes fossem capazes de compreender ainda mais o contexto individual e coletivo, preparando um plano terapêutico mais eficaz não somente no que se refere ao adoecimento mental, mas ao cuidado integral do participante. ${ }^{5}$

Alguns estudos ${ }^{17,18}$ descrevem ainda que as práticas das equipes da UAPS são focadas na doença e que a educação em saúde como um instrumento de promoção de saúde, é realizada com abordagens nas quais a cultura não é tomada como referência, concluindo que o diálogo e o respeito cultural devem fazer parte da prática da Atenção Primária. Consideram ainda que a não vinculação às UAPS's dificulta o reconhecimento dos fatores de riscos, o seguimento da população e uma prática de cuidado ampliado. Por suposto, como já descrito, a interação das UAPS's com a população por intermédio do diálogo se faz exitoso e a TCI tem como alguns de seus grandes eixos a comunicação e a antropologia cultural, em que se é levado em consideração o indivíduo em toda sua essência. Com a implementação na UAPS Edmar Fujita, o acesso aos profissionais da unidade se tornou maior, bem como a demanda para consultas individuais pela médica terapeuta e pela residente em MFC.

Entende-se a TCI como um momento de troca de experiências e aprendizado ímpar, sendo capaz de abordar o ser humano do ponto de vista "biopsicossocioespiritual", já que os participantes dividem sofrimentos e angustias e falam como foram capazes de seguir diante do problema, em seu âmbito econômico, religioso, social e psicológico, o que aborda de maneira holística o sofrimento descrito ao longo de cada sessão. A forma como cada problema é trazido ajuda a tecer redes de apoio com a comunidade e a UAPS em uma perspectiva sem preconceitos. De fato, com a implementação, alguns usuários da unidade que participaram estavam mais abertos para contar seu contexto pessoal e familiar, corroborando para uma melhor percepção daquele indivíduo pelas profissionais de saúde. Assim, numa possível abordagem individual no consultório a construção de um melhor plano terapêutico era facilitada. ${ }^{19}$
Considera-se a pesquisa sobre TCI relativamente nova no Brasil e a maioria de seus trabalhos publicados tem uma abordagem qualitativa. Os estudos concluem que tal prática reforça as redes de apoio existentes, cria espaços de inclusão e valoriza a diversidade, resgatando a herança cultural e a história pessoal do sujeito. Também vem sendo mencionada como uma nova tecnologia de cuidado em saúde mental, sendo instrumento de intervenção psicossocial. Tais análises corroboram com o que se tem observado após implementação da TCI na UAPS Edmar Fujita. O fato de as pesquisas serem majoritariamente qualitativas pode acontecer porque ao longo das sessões o caráter subjetivo se torna mais evidente, o número de participantes é variável, o que dificulta a coleta de dados quantitativos. Porém, deve-se deixar claro que, mesmo em sessões com poucos participantes, a contribuição no cuidado à saúde do participante se faz presente. ${ }^{14,20}$

De acordo com algumas publicações, a TCI também vem contribuindo para reverter o quadro de adoecimento provocado pela síndrome da miséria psíquica (situações de estresse contínuo, desesperança, abandono e insegurança), pois há uma ampliação de ações preventivas e promocionais. , $^{3,14,1620}$ Como já descrito no presente artigo, observou-se aumento do vínculo com a equipe promotora, bem como facilitação na coordenação do cuidado à saúde, o que potencializa a prevenção e promoção de saúde no local em que a TCI é implantada.

Ao longo das sessões realizadas pela UAPS descrita, considera-se que os objetivos da TCI propostos por seu fundador $^{12}$ vêm sendo alcançados continuamente, porém é provável que pelo fato de uma das terapeutas ser médica, alguns participantes, moradores ou não da comunidade, não comparecem às sessões pelo que a TCI propõe, mas para criar vínculo com a profissional individualmente, o que não pode deixar de ser valorizado, afinal a relação com a médica se torna mais estreitada e o acesso à UAPS é ampliado.

Acerca dos questionamentos levantados durante a implementação da TCI, estudos já descrevem como necessário adquirir competências de cuidado em saúde mental na prática diária dos profissionais, principalmente da Atenção Primária, os quais devem considerar a subjetividade, a singularidade e a visão de mundo do usuário no processo de cuidado integral à saúde, além de possuir o domicílio como espaço terapêutico e a comunidade como ambiente para promoção à saúde. Nessa instância, já se recomenda a TCI como tecnologia de cuidado na Atenção Primária., 3,5,

Tendo em vista a implementação da TCI como instrumento promissor para as UAPS, com a qual o participante é estimulado a se reconhecer como responsável por sua própria saúde e pela saúde da comunidade, foram observadas melhorias do vínculo com o serviço da UAPS Edmar Fujita, facilitando o trabalho da equipe promotora, bem como contribuindo para a satisfação do participante frente ao serviço de saúde. Diante disso, recomenda-se tal prática como uma ferramenta de cuidados nos programas de apoio à saúde mental e integral, sendo uma tecnologia de baixo custo que aumenta o poder de resolutividade da Atenção Primária. 


\section{REFERÊNCIAS}

1. Campos MO, Rodrigues JF Neto. Qualidade de vida: um instrumento para promoção de saúde. Revista Baiana Saúde Pública. 2008;32(2):232-40.

2. Vieira FS, Benevides RP. O Direito à saúde no Brasil em tempos de crise econômica, ajuste fiscal e reforma implícita do estado. Revista de Estudos e Pesquisas sobre as Américas. 2016;10(3):1-28.

3. Jatai JM, Silva LM. Enfermagem e a implantação da terapia comunitária integrativa na estratégia saúde da família: relato de experiência. Rev Bras Enferm. 2012;65(4):691-5.

4. Carvalho MN, Gil CR, Costa EM, Sakai MH, Leite SN. Necessidade e dinâmica da força de trabalho na atenção básica de saúde no Brasil. Cien Saude Colet. 2018;23(1):295-302.

5. Souza AJ, Matias GN, Gomes KF, Parente AC. A saúde mental no Programa de Saúde da Família. Rev Bras Enferm. 2007;60(4):391-5.

6. Rocha IA, Sá AN, Braga LA, Ferreira MO Filha, Dias MD. Terapia comunitária integrativa: situações de sofrimento emocional e estratégias de enfrentamento apresentadas por usuários. Rev Gaucha Enferm. 2013;34(3):155-62.

7. Moura SG, Ferreira MO Filha, Moreira MA, Simpson CA, Tura LF, Silva AO. Representações sociais sobre terapia comunitária integrativa construídas por idosos. Rev Gaúcha Enferm. 2017;38(2):e55067.

8. Tófoli LF, Fortes S. Apoio matricial de saúde mental na atenção primária no município de Sobral, Ce: o relato de uma experiência. Sanare. 2005;6(2):34-42.

9. Barros NF. Política nacional de práticas integrativas e complementares no SUS: uma ação de inclusão. Cienc Saúde Coletiva. 2006;11(3):850.

10. Padilha CS, Oliveira WF. Terapia comunitária: prática relatada pelos profissionais da rede SUS de Santa Catarina, Brasil. Interface. 2012;16(43):1069-84.

11. Fischborn AF, Machado J, Fagundes NC, Pereira NM. A Política das práticas integrativas e complementares do SUS: o relato de experiência sobre a implementação em uma unidade de ensino e serviço de saúde. Cinergis. 2016;17(4 Supl 1):358-63.
12. Barreto AP. Terapia comunitária passo a passo. Fortaleza: Gráfica LCR; 2005.

13.Andrade FB. A terapia comunitária como instrumento de inclusão da saúde mental na atenção básica: avaliação da satisfação dos usuários [Dissertação]. João Pessoa: Universidade Federal da Paraíba; 2009 [Acesso em: 03 jan 2018]. 141p. Disponível em: http://www.ccs.ufpb.br/ppgeold/dissertacoes2009/fabiabarbosa. pdf

14. Rangel CT, Miranda FA, Oliveira KK. A terapia comunitária integrativa e a enfermagem: o fenômeno e seus contextos[Internet]. Revista de Pesquisa Cuidado é Fundamental. 2016[acesso em: 10 dez 2017];8(1):3770-9. Disponível em: http://seer.unirio.br/index.php/ cuidadofundamental/article/view/3997

15. Melo SP, Ribeiro RL, Costa AL, Urel DR. Repercussões da terapia comunitária integrativa nas pessoas doentes renais durante sessão de hemodiálise [Internet]. Revista de Pesquisa Cuidado é Fundamental. 2015[acesso em: 10 dez 2017];7(2):2200. Disponível em: http://seer. unirio.br/index.php/cuidadofundamental/article/view/2841

16. Guimarães FJ, Ferreira MO Filha. Repercussões da terapia comunitária no cotidiano de seus participantes [Internet]. Revista Eletrônica de Enfermagem. 2006[acesso em: 11 dez 2017];8(3):40414. Disponível em: http://www.fen.ufg.br/revista/revista8_3/ v8n3a11.htm

17. Teixeira C, Silva CC, Bernardes GC, Sá NP, Prado RS. O vínculo entre usuários e equipes em duas unidades de saúde da família em um município do estado do Rio de Janeiro. Rev APS. 2013;16(4):444-54.

18. Silocchi C, Junges JR. Equipes de atenção primária: dificuldades no cuidado de pessoas com doenças crônicas não transmissíveis. Trab Educ Saúde. 2017;15(2):599-615.

19. Barreto AP. Quando a boca cala, os órgãos falam: desvendando as mensagens dos sintomas. Fortaleza: LCR; 2014.

20. Ferreira MO, Lazarte R, Barreto AP. Impacto e tendências do uso da terapia comunitária integrativa na produção de cuidados em saúde mental [Internet]. Revista Eletrônica de Enfermagem. 2015[acesso em 11 dez 2017];17(2):172-3. Disponível em: http://revistas.ufg.br/ index.php/fen/article/view/37270

\section{Como citar:}

Forte MP, Pereira AS. A implementação da Terapia Comunitária Integrativa em uma unidade de saúde e seu auxílio no cuidado integral: um relato exitoso. Rev Med UFC. 2019 out-dez;59(4):34-38. 